\title{
Carl von Rokitansky, el Linneo de la anatomía patológica
}

Carlos Ortiz-Hidalgo*

Clínica Fundación Médica Sur, Departamento de Anatomía Patológica, Ciudad de México, México

\section{Resumen}

Carl von Rokitansky fue una de las figuras más importantes en la anatomía patológica y el responsable, en parte, del renacimiento de Viena como centro de la medicina a mediados del siglo XIX. Nació en la actual Hradec Králové, estudió medicina en Praga y Viena y se graduó en 1828. Tuvo gran influencia de los estudios de anatomía, embriología y patología de Andral, Lobstein y Meckel. En la escuela de Viena fue asistente de anatomía patológica de Johann Wagner y se convirtió en profesor de anatomía patológica, donde permaneció hasta cuatro años antes de su muerte. Rokitansky hizo énfasis en correlacionar la sintomatología del enfermo con los cambios post mortem. Es posible que haya tenido acceso a entre 1500 y 1800 cadáveres al año para que pudiera realizar 30000 necropsias; además, revisó varios miles más de autopsias. En Handbuch der Pathologischen Anatomie, publicado entre 1842 y 1846, realizó numerosas descripciones: de la neumonía lobular y lobular, endocarditis, enfermedades de las arterias, quistes en varias vísceras, diversas neoplasias y de la atrofia aguda amarilla del hígado. Con su brillante labor de patología macroscópica, Rokitansky estableció la clasificación nosológica de las enfermedades, por lo cual Virchow lo llamó "el Linneo de la anatomía patológica".

PALABRAS CLAVE: Carl von Rokitansky. Historia de la patología. Nueva escuela de medicina de Vienna.

\section{Carl von Rokitansky, the Linné of pathological anatomy}

\begin{abstract}
Carl von Rokitansky was one of the most important figures in pathological anatomy, and was largely responsible for the resurgence of Vienna as the great medical center of the world in the mid-19 century. He was born in current Hradec Králové, studied medicine in Prague and Vienna and was graduated in 1828. He was greatly influenced by the anatomy, embryology and pathology studies of Andral, Lobstein and Meckel. At the Vienna School, he was Johann Wagner pathological anatomy assistant and became a pathology professor, where he remained until four years before his death. Rokitansky emphasized the importance of correlating patient symptoms with postmortem changes. It is possible that he had access to between 1,500 and 1,800 cadavers annually to be able to perform 30,000 necropsies; in addition, he reviewed several thousand more autopsies. In Handbuch der pathologischen Anatomie, published between 1842 and 1846, he made numerous descriptions: lobar and lobular pneumonia, endocarditis, diseases of the arteries, cysts in several viscera, various neoplasms and acute yellow atrophy of the liver. With his brilliant work on gross pathology, Rokitansky established the nosological classification of diseases, for which Virchow named him "the Linné of pathological anatomy".
\end{abstract}

KEY WORDS: Carl von Rokitansky. History of pathology. New Vienna Medical School.

Correspondencia:

${ }^{*}$ Carlos Ortiz-Hidalgo

E-mail: ortizhidalgocarlos@gmail.com

0016-3813/@ 2020 Academia Nacional de Medicina de México, A.C. Publicado por Permanyer. Este es un artículo open access bajo la licencia CC BY-NC-ND (http://creativecommons.org/licenses/by-nc-nd/4.0/).
Fecha de recepción: 09-01-2020

Fecha de aceptación: 04-02-2020
Gac Med Mex. 2020;156:595-603

Disponible en PubMed www.gacetamedicademexico.com
lo open access bajo la licencia CC 
El profesor mediocre dice. El profesor bueno explica. El profesor superior demuestra. El profesor excelente inspira. William Arthur Ward (1921-1994)

\section{Introducción}

Entre 1860 y 1910, Viena se convirtió en la capital cultural de Europa y tenía gran afluencia de talentos, lo que resultó en un florecimiento cultural e intelectual asombroso. Ahí se encontraban personajes como Freud, Mahler y Klimt, entre otros. ${ }^{1}$ En particular, el conocimiento del cuerpo humano que plasma Klimt en sus pinturas fue por presenciar varias disecciones en cadáveres con Rokitansky. Rokitansky, Škoda y Hebra fueron los pilares de la llamada "nueva escuela de Viena", centro académico de renombre mundial. Škoda mejoró los métodos clínicos de diagnóstico, Hebra sobresalió por sus habilidades diagnósticas y terapéuticas en dermatología y las contribuciones de Rokitansky fueron primordiales para el desarrollo de la anatomía patológica. ${ }^{2}$

Para finales del siglo XIX se vivía una gran hostilidad por parte de la comunidad médica hacia la práctica de autopsias. Tomó el trabajo incansable de un extraordinario hombre, Carl Rokitansky, para cambiar el rumbo de la medicina, gracias al conocimiento que pudo obtener del estudio de autopsias. Rokitansky es una de las figuras más importantes en la anatomía patológica y fue quien protagonizó una auténtica revolución académica, estableciendo la llamada "nueva anatomía patológica”. ${ }^{3}$

\section{Vida y obra de Rokitansky}

Carl Joseph Wenzl Prokop Rokitansky (en checo Karel Rokytanský, 1804-1878) nació en Königgrätz, hoy Hradec Králové, actual República Checa, en la casa número 42 de la plaza mayor, cerca de la Catedral del Espíritu Santo, en cuya fachada existe una placa en su honor que fue develada un año después de su muerte (Figura 1). Estudió la escuela elemental en Königgrätz y a los 14 años se trasladó a Praga a estudiar filosofía en la Univerzita Karlova, atraído seguramente por la gran admiración que le causaba el filósofo y epistemólogo Bernard Bolzano (1741-1848), seguidor de Immanuel Kant (1724-1804); más tarde, Rokitansky fue admirador de la filosofía de Schopenhauer., ${ }^{4,5}$

En 1821, Rokitansky inició sus estudios de medicina en Praga, donde cursó anatomía con Jan Evangelista Purkyně (1787-1869) y completó estudios de zoología, mineralogía, botánica, fisiología, química, farmacia y patología general. ${ }^{6}$ De acuerdo con lo informado por Steiner, Rokitansky no fue un alumno brillante pues obtuvo la calificación de $\mathrm{E}$ (excelente) solo en el curso de anatomía y en el de cirugía teórica. ${ }^{4} \mathrm{Al}$ cumplir los 20 años de edad, y después de tres años de estudio en Praga, se marchó a Viena, pues decía que sus profesores no estaban interesados en enseñar a los estudiantes; en Viena encontró un ambiente académico mucho más estimulante. Terminó medicina en 1828 con la tesis De varioloide vaccinica, en torno a la enfermedad que había sido una plaga en Europa hasta finales del siglo XVIII.7,8

Durante su carrera le interesaron particularmente los textos de Johann F. Lobstein (1777-1835), Friedrich S. Meckel el joven (1781-1833) y Gabriel Andral (17971876), por lo que decidió dedicarse a la anatomía patológica. No practicó medicina clínica, pero convivió con renombrados médicos de la época como Josef Škoda (1805-1881) y Ferdinand von Hebra (1816-1880), entre otros (Figura 2).

Unos meses antes de terminar la carrera, Rokitansky aceptó el puesto sin remuneración de prosector en el área de autopsias (leichenhaus) del Hospital General de Viena (Wiener Allgemeines Krankenhaus). Ahí trabajo con el profesor Johann Wagner (1800-1833), a quien ayudó a realizar la autopsia de Beethoven, el 27 de marzo de $1827 .^{5,9} \mathrm{~A}$ la prematura muerte de Wagner en 1832 (murió a los 33 años por tuberculosis), Rokitansky fue nombrado profesor asociado y encargado del servicio de anatomía patológica, convirtiéndose así en el primer patólogo de tiempo completo. En 1844 fue designado profesor y jefe de Anatomía Patológica y la materia de Patología fue establecida en el currículo médico (la Universidad de Viena fue la segunda en establecer la plaza de profesor de Anatomía Patológica; la primera fue la de Estrasburgo en 1791); en 1849 ocupó la dirección de la Facultad de Medicina de Viena, en 1852 fue electo rector magnificus y 10 años después fue designado asesor médico (Medizinalreferent) de cultura e instrucción. Ingresó a la Academia de Ciencias en 1848 y a partir de 1850 y durante 28 años fungió como presidente de la Sociedad Médica de Viena. En 1874 fue distinguido con el título de barón (Freiherr) (Figura 3).

\section{La experiencia de Rokitansky en las autopsias}

El problema de la anatomía patológica en el tiempo en que Rokitansky comenzaba su vida profesional fue que esta disciplina era vista en muchas ocasiones sin 


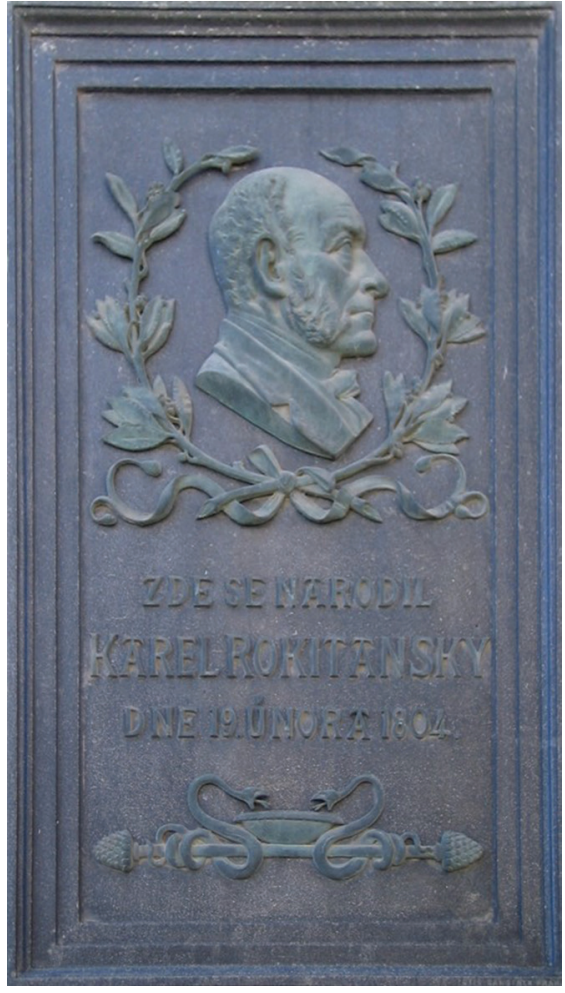

Figura 1. Placa conmemorativa que se encuentra en el lugar donde nació Rokitansky en la ciudad de Hradec Králové, en la República Checa. El texto checo dice "Karel Rokitansky nació aquí el 19 de febrero de 1804". Este bajorrelieve fue realizado por el escultor checo Josef Václav Myslbek (1848-1922) y se develó en 1879, un año después de la muerte de Rokitansky.

importancia. Para entonces se trataba de explicar los cambios anatómicos en términos de los hallazgos clínicos, es decir, el hallazgo anatómico era interpretado de acuerdo con un dato clínico particular. El célebre fisiólogo francés François Magendie (17831855) pensaba que la anatomía patológica tenía poca utilidad y hacia 1839 afirmó:

\footnotetext{
...le doy muy poco valor al examen minucioso de las huellas dejadas en los órganos a través de esa búsqueda que pomposamente ha sido llamada Anatomía Patológica. Las lesiones encontradas en las autopsias son frecuentemente producidas después de la muerte y consecuentemente el plan hasta ahora que se ha seguido en estas investigaciones, es una falacia y únicamente puede conducir a información vaga y al error...
}

A este escenario se enfrentó Rokitansky y quizás una de las aportaciones más importante de este distinguido patólogo fue que pudo convencer, no sin menudos enfrentamientos académicos, a los médicos clínicos de la época de que aceptaran que la clínica tenía que ser interpretada a partir de los hallazgos anatómicos. $^{2-4}$ Bien había dicho Giovanni Battista Morgagni (1682-1771) que los síntomas son "el llanto de los órganos que sufren". Rokitansky estudiaba las alteraciones morfológicas y reconstruía el proceso de la enfermedad, correlacionando los hallazgos con la sintomatología que en vida había tenido el paciente, y trataba de indagar la etiología y patogenia de la enfermedad, así como los resultados de la terapia empleada. Esto hizo acercar a los médicos clínicos a la sala de autopsias para aprender de las lesiones anatómicas, asentando así la correlación anatomoclínica y las bases de las sesiones anatomopatológicas actuales. Todo esto generó molestia entre muchos médicos hasta el punto de exigir que retiraran la licencia para practicar medicina a Rokitansky. ${ }^{10,11}$

De 1834 a 1862, Rokitansky trabajó en la "vieja sala de autopsias" (Figura 4), un lugar muy mal acondicionado para el trabajo, con poca iluminación y mala ventilación (los estudiantes la llamaban "la cabaña miserable"), por lo que es admirable la cantidad y calidad del trabajo realizado bajo tales circunstancias. No fue sino hasta 1862, durante un congreso médico en Viena, que médicos alemanes criticaron fuertemente al Imperio por las condiciones de las instalaciones lamentables en que Rokitansky trabajaba, y fue como consecuencia de esto la apertura meses después del nuevo Instituto de Patología en Viena..$^{11}$ Ahí, el trabajo de anatomía patológica fue monumental.

Por un decreto firmado en 1753 por María Teresa I de Austria (1717-1780), todo paciente que moría en el Hospital General de Viena pasaba al Instituto de Patología para practicarle la autopsia, por lo que es muy probable que Rokitansky haya tenido acceso a unos 1800 cadáveres al año. Rokitansky realizó 30000 autopsias en toda su vida y se tienen los protocolos escritos con su puño y letra. ${ }^{11,12}$ Además, supervisó cerca de 40000 autopsias más, por lo que si consideramos que realizó la última autopsia a los 62 años y comenzó sus estudios de patología a los 23 años, 30000 autopsias representan de dos a tres autopsias diarias, los 365 días del año, durante 38 años. El primer protocolo tiene fecha del 23 de octubre de 1827 y el número 30000 tiene fecha de $1866.1^{11-13} Y$ por si todo este trabajo fuera poco publicó más de 400 artículos y 20 libros.

En muchos de sus artículos y en su fenomenal libro de tres volúmenes titulado Handbuch der pathologischen Anatomie, que apareció publicado en 1842, Rokitansky mostró los hallazgos anatómicos de diversas enfermedades (Figura 5). Describió magistralmente numerosas alteraciones anatómicas de diversos padecimientos, como la obstrucción intestinal por hernia interna, la 


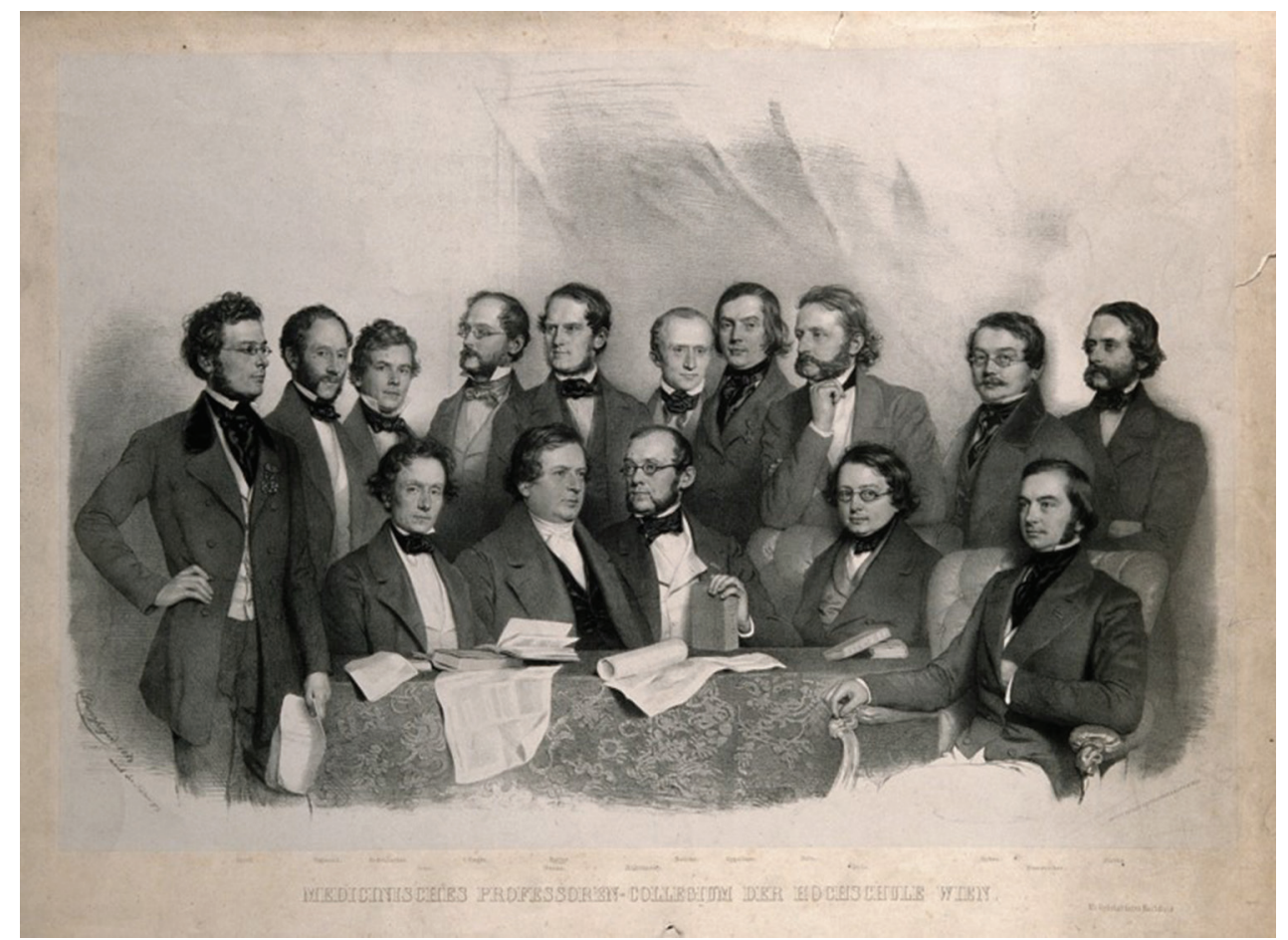

Figura 2. Quince miembros de la Universidad de Viena en 1853. Parados de izquierda a derecha: Josef Hyrtl, Karl Ludwig von Sigmund, Josef Redtenbacher, Franz Unger, Carl Haller, Ernst Wilhelm von Brücke, Johann von Oppolzer, Theodro Helm, Ferdinand Ritter von Hebra, Johann Dlauhy. Sentados de izquierda a derecha: Franz Schuh, Anton von Rosas, Karl von Rokitansky, Josef Skoda, Johann H. G. von Dumreicher. Litografía de Auguste Prinzhofer (1817-1885). Disponible en el sitio web Wellcome Collection.
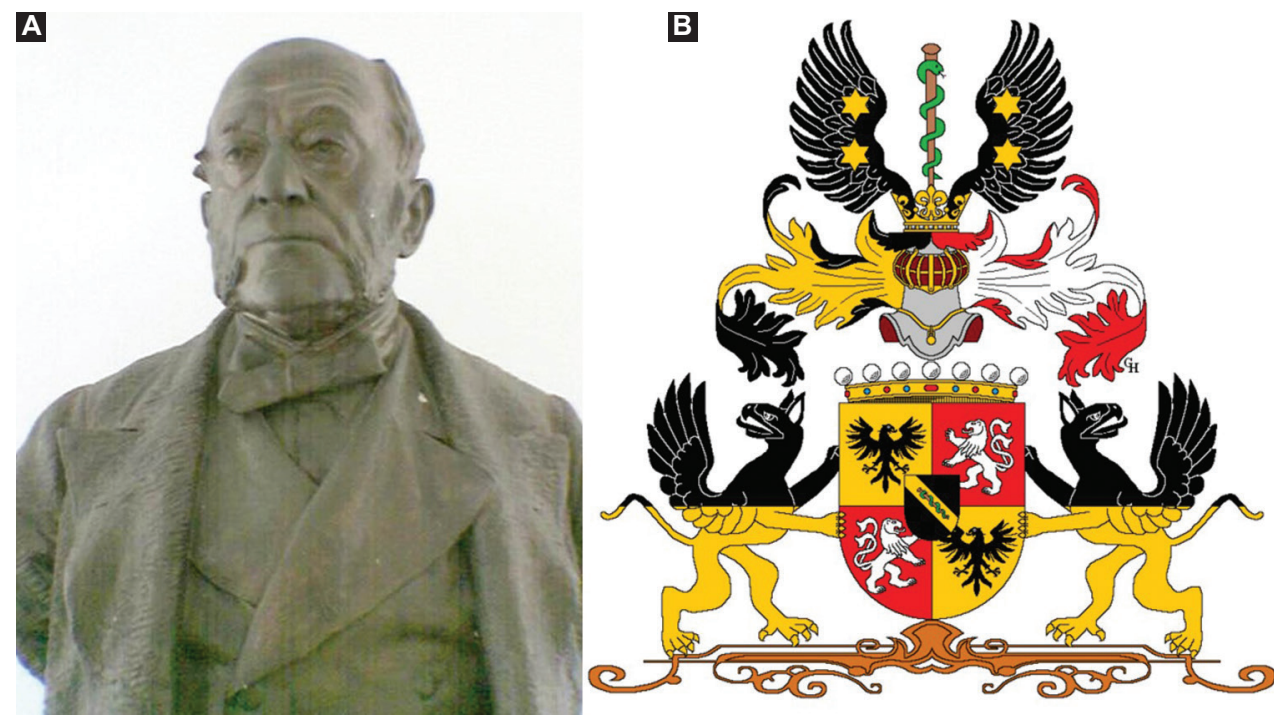

Figura 3. A) Busto de Carl Rokitansky que se encuentra en los pasillos del Departamento de Patología de Graz en Viena (fotografía tomada por el autor). B) Escudo otorgado a Rokitansky cuando lo hicieron barón (Freiherr) en 1874.

dilatación aguda del estómago, diversos tumores de la vesícula biliar, el síndrome de la arteria mesentérica superior y propuso que la causa de esta era la obstrucción de la tercera porción del duodeno por compresión de la arteria mesentérica (síndrome de Rokitansky).
En el Handbuch, Rokitansky describió por primera vez la obstrucción de las venas suprahepáticas por trombos en pacientes cirróticos, posteriormente observada por George Budd (1808-1882) en 1845 y por Hans Chiari (1851-1916) en 1889, que hoy 
Tabla 1. Epónimos con el nombre de Rokitansky y descripciones originales

Síndrome de la arteria mesentérica superior (síndrome de Rokitansky-Wilkie)

Síndrome de Mayer-Rokitansky-Küster-Hauster.

Divertículo de Rokitansky

Estenosis pulmonar de Rokitansky

Senos de Rokitansky-Aschoff

Úlcera de Rokitansky-Cushing

Síndrome de Rokitansky-Maude Abbott

Nódulo de Rokitansky (en los teratomas ováricos)

Sarcoma de Rokitansky (sarcoma filodes de mama)

Incisión de Rokitansky

Endometriosis de Rokitansky

Periarteritis nodosa de Rokitansky (diátesis aneurismática)

Síndrome de von Rokitansky

Lóbulos de Rokitansky

Enfermedad de Rokitansky (síndrome de Budd-Chiari)
Pinzamiento aortomesentérico debido a compresión intermitente de la tercera porción del duodeno, por nacimiento anómalo de la arteria mesentérica superior

Agenesia de vagina con amenorrea primaria

Divertículo por tracción del esófago

Estenosis pulmonar. Defecto septal subaórtico de la posición lateral derecha de la aorta

Invaginaciones de la mucosa hasta la muscular o serosa

Úlceras que a veces acompañan lesiones severas del sistema nervioso central y afectan esófago, estómago y duodeno

\section{Comunicación interaurículo-ventricular}

Nódulo interno compuesto por tejido embrionario maduro (generalmente diente) en un teratoma quístico de ovario.

Tumor fildeos de la mama

Incisión en la reflexión peritoneal lateral para tener acceso al retroperitoneo

Endometriosis ovárica

Periarteritis nodosa

Transposición corregida de las grandes arterias

Lóbulos pulmonares supernumerarios, generalmente aislados de tejido pulmonar y separados tanto del árbol traqueobronquial como del sistema vascular pulmonar

Trombosis de las venas suprahepáticas conocemos como síndrome de Budd-Chiari. ${ }^{6,14}$ Además, describió las dilataciones diverticulares de la mucosa vesical (senos de Rokitansky-Aschoff), la dilatación aguda del esófago, la atrofia aguda amarilla del hígado, los infartos hepáticos, la sinovitis villonodular, la poliarteritis nodosa, la neumonía lobar e intersticial, los divertículos traqueales, la ruptura espontánea de la aorta, la estenosis pulmonar (estenosis de Rokitansky), diversas malformaciones congénitas del corazón, la ausencia de vagina con amenorrea primaria/agenesia parcial mulleriana (síndrome de Mayer-Rokitansky-KüsterHauser; posiblemente Eva Braun, la mujer de Hitler, tuvo este síndrome), ${ }^{15}$ el tumor filodes mamario (cistosarcoma de Rokitansky), la presencia de un tubérculo dentro de un teratoma ovárico quístico que se proyecta hacia la luz del quiste (nódulo de Rokitansky), que correspondía a tejido adiposo, pelos, dientes o hueso; el útero doble, el bocio nodular, el adenoma pleomórfico de la glándula parótida, los ovarios poliquísticos, la endometriosis ovárica y la adenomiosis uterina; además, realizó la

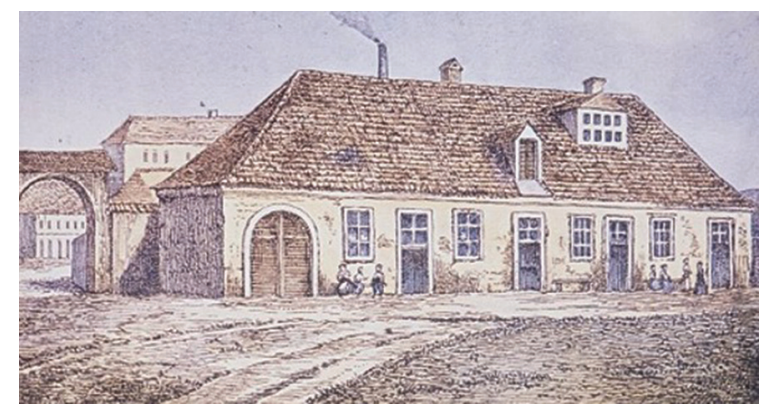

Figura 4. Lacasa donde Rokitansky realizaba autopsias. Fuente:Center for Forensic Medicine, Medical University of Vienna [sitio web]. En: https://www.meduniwien.ac.at/hp/en/forensic-medicine/ general-information/history/

primera descripción de espondilolistesis,,$^{16}$ entre muchas otras contribuciones ${ }^{3}$ (Tabla 1). Rokitansky acuñó también los términos "melanoma pigmentado", "hamartoma" y "osteoide" y es a él a quien se atribuyen las descripciones metafóricas "vómito en granos de café", correspondiente a la hematemesis, o "diarrea en puré de guisantes", que se presenta en algunas diarreas infecciosas. . $^{5,17,18}$ 


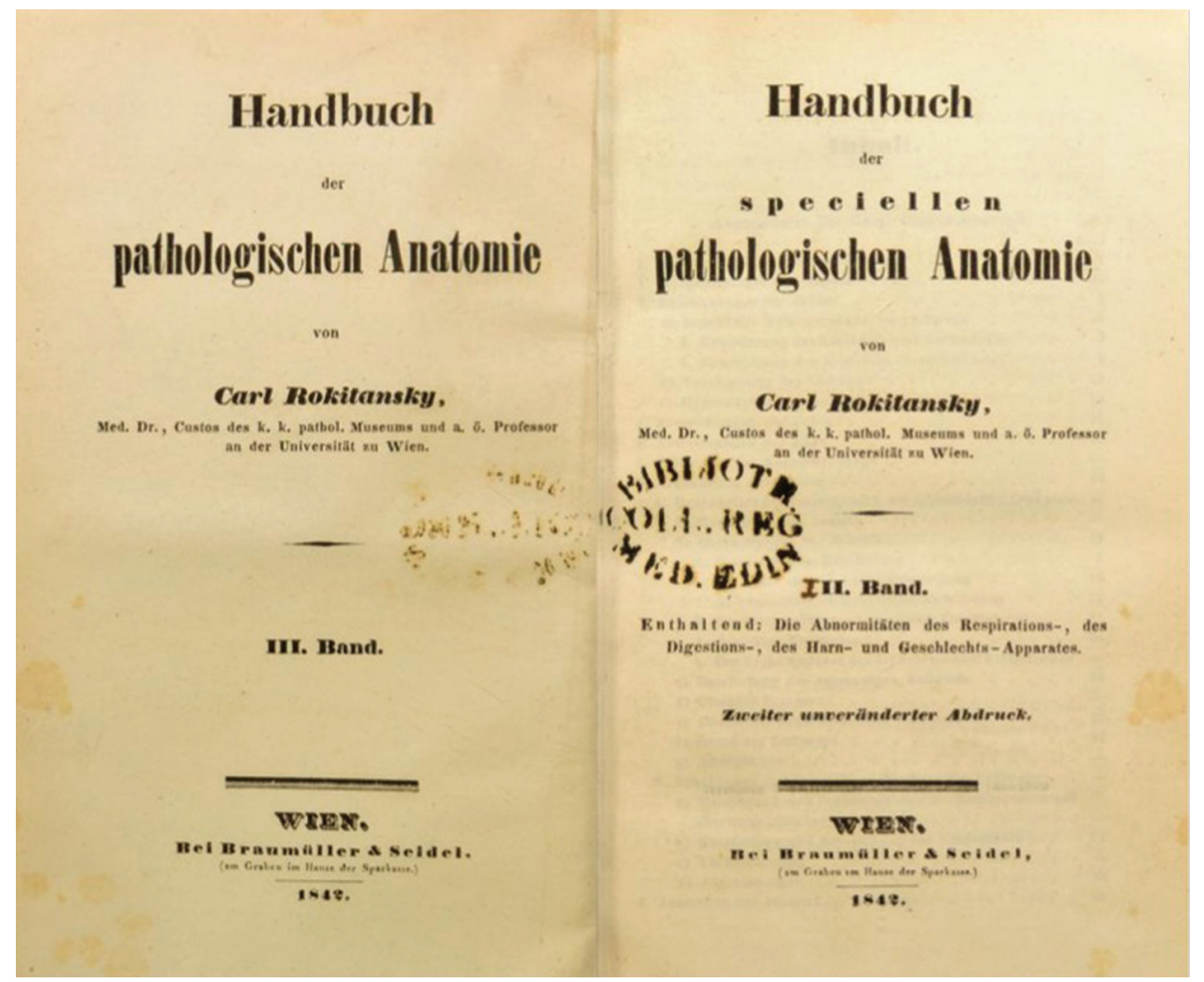

Figura 5. Portada del libro Handbuch der Speziellen Pathologischen Anatomie. Este libro le valió a Rokitansky la Cruz de Honor de Austria por la Ciencia y el Arte de Primera clase "litteris et Artibus" (Österreichisches Ehrenkreuz für Wissenschaft und Kunst 1. Klasse "Litteris et Artibus").

El Handbuch le valió a Rokitansky la Cruz de Honor de Austria por la Ciencia y el Arte de Primera Clase "Litteris et Artibus" (Österreichisches Ehrenkreuz für Wissenschaft und Kunst 1. Klasse "Litteris et Artibus"). ${ }^{19}$

Otro extraordinario libro de Rokitansky fue el publicado en 1875, titulado Defectos en el septo del corazón (Die Defekte der Scheidewände des Herzens), donde imprimió sus observaciones acerca de las cardiopatías congénitas y presentó su teoría en cuanto a la transposición que deriva de la desviación del septo aórtico. ${ }^{20}$ Rokitansky demostró, además, detalles precisos de la anatomía del tabique interauricular e interventricular y fue el primero en describir las diferencias entre los defectos del ostium primum y ostium secundum. El libro representa el complemento del que había publicado años antes, en 1852, titulado Sobre algunas de las más importantes enfermedades de las arterias (Ueber Einige der Wichtigsten Krankheiten der Arterien). ${ }^{21}$

Por lo que respecta al cáncer, designó como "cáncer epitelial" aquel en el cual, al examen microscópico en algunos tumores de piel, labio, escroto y prepucio, se podían identificar células análogas en todos los aspectos a las células epidérmicas normales. Con ello dio la sugerencia del origen epitelial del cáncer, mientras otros patólogos, incluyendo al renombrado Virchow, erróneamente consideraban al cáncer como de origen del tejido conectivo.

Conforme pasó el tiempo y su fama aumentó, Rokitansky adquirió numerosos ayudantes que llegaban de varios puntos de Europa. El Institut für Pathologische del Hospital General de Viena se convirtió en centro importante de la medicina austriaca y dejó numerosos alumnos como Hans Chiari (18511916), Ferdinand von Hebra (1816-1880), Ignác Fülöp Semmelweis (1881-1865), Carl Wedl (1815-1891, maestro de Moritz Kaposi), el anatomista Josef Engel (1816-1899) y el discípulo favorito de Rokitansky, Hans Kundrat (1845-1893), quien acuñó el término "linfosarcoma" (enfermedad de Kundrat) y fue su sucesor en las dirección del Laboratorio de Anatomía Patológica. ${ }^{22}$

Es de particular interés la relación que tuvo la escuela de Rokitansky con Semmelweis, quien fue nombrado asistente en una de las clínicas de maternidad del Hospital General de Viena y fue ahí donde 

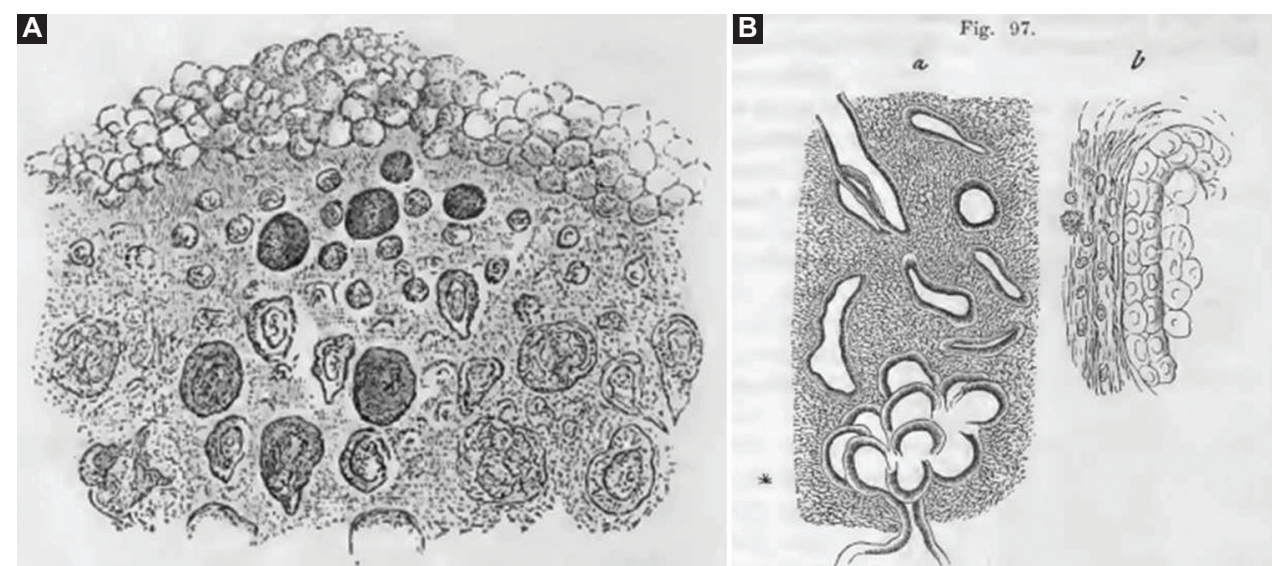

Figura 6. Algunas observaciones microscópicas que Rokitansky ilustró en su libro Handbuch der Speziellen Pathologischen Anatomie. A) En el dibujo señala la "metamorfosis coloide" (amiloide) en un paciente con parálisis. B) Dibujo de un corte histológico de lo que llamó "cistosarcoma mamario", hoy conocido como tumor filoides o cistosarcoma de Rokitansky.

observó que los alumnos de Rokitansky después de hacer autopsias iban a atender partos y algunas mujeres morían de sepsis puerperal. Pensó que los estudiantes transportaban algún tipo de "material putrefacto" y la solución fue sencilla; indicó que todo el personal se lavara las manos en una solución de cloro antes de examinar a las embarazadas y así, casi de inmediato, la mortalidad fue mucho menor. ${ }^{22}$

Regresando a la vida académica de Rokitansky, al ver que muchos cadáveres no tenían alteraciones macroscópicas evidentes, desarrolló la hipótesis hemato-humoral Krasenlehre..$^{13}$ De acuerdo con esta, la sangre era la responsable del proceso de enfermedad por el resultado de la falta de balance entre la fibrina y la albúmina. Cuando los elementos (a los cuales llamaba blastema) salían de los vasos y pasaban al intersticio, tenían la capacidad de coagulación y solidificación y de formar componentes tisulares.

Rokitansky fue severamente criticado por el joven Virchow (que entonces tenía 25 años). ${ }^{13}$ Rudolf Ludwig Karl Virchow (1821-1902) no fue su alumno, sino uno de sus más severos críticos. Rokitansky atribuía la causa de la enfermedad a cambios en las propiedades químicas de las proteínas de la sangre (fibrina y albúmina), que resultaban de la formación local de "blastema", que a su vez se transformaban en algunas de las lesiones que él veía en la mesa de autopsia. Para Virchow, a pesar de la admiración que tenía hacia Rokitansky, esta "teoría hemato-humoral" era un enorme anacronismo (ungeheure anachronismus). En su famosa revisión publicada en diciembre de 1846 (en dos suplementos de la revista médica prusiana Preussische Medizinal-Zeitung), lo inculpó de proponer una teoría sin evidencia científica. Rokitansky aceptó la crítica y muchos de los conceptos censurados por Virchow fueron modificados en la nueva edición de su libro publicado en 1855 titulado Lehrbuch der pathologischen Anatomie. ${ }^{23}$

Es verdad que Rokitansky prefirió el examen macroscópico al microscópico, pero existe un considerable número de observaciones microscópicas que se encuentran ilustradas en sus libros (Figura 6). En su autobiografía describe que el microscopio lo utilizaba mayormente cuando sospechaba cáncer; sin embargo, es verdad que no le dio la misma importancia que le dio Virchow. ${ }^{8}$

En relación con su vida personal, se le atribuía una actitud pesimista y depresiva, con preocupaciones económicas constantes, lo que lo obligó a impartir clases particulares de anatomía y patología. Adolf Kussmaul (1822-1902), quien trabajó con Rokitansky durante una temporada, lo calificó como de carácter triste y melancólico, fundamentalmente pesimista y sombrío, y esa posiblemente fue la razón de su afición por las lecturas filosóficas de Schopenhauer. ${ }^{24}$ Rokitansky llegó a decir que....,5,24

En la vida animal y en especial la humana, el sufrimiento es mucho más prevalente que la alegría, y la alegría no es nada más que un deseo satisfecho, un sufrimiento desahogado, una negación del mismo...

Sin embargo, otros biógrafos dicen que este era solo un pequeño rasgo de su personalidad y que él fue básicamente feliz. ${ }^{6,10}$ En 1834, al cumplir los 30 años de edad, se casó con la cantante de ópera María Ana Weiss, quien heredó su talento artístico a dos de 
sus hijos, Hans (1835-1909) y Viktor (1836-1896), y los otros dos, Karl (1839-1898) y Prokop (1843-1928) fueron médicos. En realidad, la pareja tuvo siete hijos, tres de los cuales murieron a edad temprana. Rokitansky solía decir, en relación con sus hijos que "die Einen heilen, die Anderen heulen" (unos curan y otros aúllan). Los domingos, Rokitansky invitaba a sus alumnos a su casa para tomar té con pastelillos y hablar de temas diversos de cultura y escuchar cantar a su esposa e hijos. ${ }^{5,24}$

Rokitansky fue el primer presidente de la Sociedad de Antropología y en 1863 publicó dos ensayos concernientes a la organización universitaria (Zeitfragen, Betreffend die Universität, mit Besonderer Beziehung auf Medecin y Die Conformität der Universitäten mit Rücksicht auf Gegenwärtige Österreichische Zustände), en donde expresaba su convicción de que la universidad debería tener diversas facultades y que todas las universidades de Austria deberían tener un estándar académico uniforme, con un doble papel de enseñanza e investigación. ${ }^{5,23}$ Además de los libros médicos, Rokitansky escribió ensayos sobre el valor intrínseco de la mente (1867) y la solidaridad de toda la vida animal (1869)., ${ }^{5,11}$

Al celebrarse una fiesta por sus 70 años de vida, cuentan que las calles de Viena estaban adornadas, había música por todas partes y varios exalumnos llegaron de diversas partes de Europa para felicitar al "padre Roki", como solían llamarle. A Rokitansky se le entregó por parte del gobierno austriaco, un certificado de honor donde decía "...es usted la gloria de Austria y un honor de la Universidad..."

Sir William Osler (1849-1919), llamado el "padre de la medicina moderna", fue uno de los invitados a la celebración, quien la describió: $:^{11,13}$

\footnotetext{
A la hora citada, no había ya lugar en el amplio auditorio... había numerosas personas para festejar al pionero de la patología moderna. Al concluir la ceremonia se develó un hermoso busto en medio de los aplausos de la concurrencia. Se recibió a por lo menos 34 personajes de diversas partes de Europa quienes presentaron cada uno, sus felicitaciones...aproximadamente 150 estudiantes, todos con antorchas y muchos con el uniforme de sus universidades, caminaron por las calles de Viena haciendo alusión al festejo del profesor...Los habitantes de la ciudad se asomaban por sus ventanas para ver la procesión...

Rokitansky dijo:

...yo he ganado para la anatomía patológica un lugar tan importante en la ciencia alemana, que puedo designarla como una base para la ciencia de la fisiología patológica y como los cimientos de la investigación en la esfera de la medicina...
}

Tres años después de su jubilación y aquejado por bronquitis crónica, asma, dolor precordial y disnea, Rokitansky murió a los 74 años de edad, en la mañana del 23 de julio de 1878. Está enterrado en el cementerio Hernalser Friedhof de Viena, donde también está Hebra y su hijo Hans..$^{24,25}$ En la Ciudad de México, Rokitansky está pintado en el extremo superior izquierdo del segundo mural que realizó Diego Rivera en el auditorio del Instituto Nacional de Cardiología.

Rokitansky es considerado el personaje más importante en la reorientación de la medicina en Viena del siglo XIX y el iniciador de la patología moderna. Fue por todo lo anterior que Virchow lo llamó "el Linneo de la anatomía patológica". ${ }^{5}$

\section{Conflicto de intereses}

No hay conflicto de intereses en relación con el artículo que se remite para publicación.

\section{Financiamiento}

Declaro que no recibí ninguna fuente del financiamiento para esta investigación.

\section{Responsabilidades éticas}

Protección de personas y animales. Para esta investigación no se realizaron experimentos en seres humanos ni en animales.

Confidencialidad de los datos. En este artículo no aparecen datos de pacientes.

Derecho a la privacidad y consentimiento informado. En este artículo no aparecen datos de pacientes.

\section{Bibliografía}

1. Steiner I. Czech medical personalities in Vienna during the 19th century. Wien Med Wochenschr. 2020;170:268-273.

2. Lesky E. The Vienna Medical School of the 19th Century. EE. UU.: Johns Hopkins University Press; 1976.

3. Jay V. The legacy of Karl Rokitansky. Arch Pathol Lab Med. 2000;124:345-346.

4. Steiner I. Rokitansky in this Bohemian years and his relations with Jan $\mathrm{E}$. Purkyne. Wien Klin Worchenschr. 2004;116;788-791.

5. Batts RE. Intellectual development of Carl Rokitansky. En: A history of endometriosis. Reino Unido: Springer; 2011.

6. Bureš J. Karel Rokitansky. República Checa. Folia [En línea]; 2004.

7. Ferraz-de Campos FP. The dawn of modern pathology. Autops Case Rep. 2016;6:1-5.

8. Lesky E. Carl von Rokitansky: Selbstbiographie und Antrittsrede. Alemania: Österreichischen Akademie der Wissenschaften; 1960.

9. Oiseth SJ. Beethoven's autopsy revisited: a pathologist sounds a final note. J Med Biogr. 2017;25:139-147.

10. Glider SSB. Carl Von Rokitansky (1804-1878). Canad M A J. 1954;71:70-72.

11. Menne FR. Carl Rokitansky. The Pathologist. Ann Med Hist. 1925; 379;379-386.

12. Ortiz-Hidalgo C. Las 30,000 autopsias. En: Narrativa médica en los 150 años de la Academia Nacional de Medicina de México. Med Intern Mex. 2014;30:755-756. 
13. Miciotto RJ. Carl Rokitansky: a reassessment of the hematohumoral theory of disease. Bull Hist Med. 1978;52:183-199.

14. Rokitansky C. Handbuch der pathologischen Anatomie. Alemania: Braumüller \& Seidel; 1846.

15. Lundmark T. The untold story of Eva Braun, her life beyond Hitler. Createspace Independent Publishing Platform; 2011.

16. Emge LA. The elusive adenomyosis of the uterus. Its historical past and its present state of recognition. Am J Obstet Gynecol. 1962;83:1541-163.

17. Hadju S. Pathologits who attained fame without microscopy. Ann Clin Lab Sci. 2003;33:119-122.

18. Carl von Rokitansky (1804-1878). JAMA. 1963;183:1105-110.

19. Castagnoli L, Jonjic N, Rizzardi C, Melato M. Carl von Rokitansky and the Italian translation of the Handbuch der Pathologischen Anatomie: a linguistic and doctrinal dilemma. Pathologica. 2001:93:654-661.
20. Rokitansky C. Die Defekte der Scheidewände des Herzens. Austria: W. Braumüller; 1875.

21. Rokitansky C. Üeber Einige der Wichtigsten Krankheiten der Arterien. Austria: Hof- und Staatsdruckerei; 1852.

22. Jadraque PP, Carter KC. What happened at Vienna's Allgemeines Krankenhaus after Semmelweis's contract as assistant in the first maternity division was terminated? Epidemiol Infect. 2017;145:2144-2151.

23. Rokitansky C. Lehrbuch der pathologischen Anatomie. Volumen 1. Braumüller \& Seidel; 1855.

24. Weyeres W. Carl Rokitansky: his life, work, and contributions to dermatopathology on the occasion of his 200th birthday. Am J Dermatopathol. 2004;26:431-438.

25. Sedivy R. Rokitansky's diseases and cause of death: a short pathological and historical discourse. Wien Med Wochenschr. 2010;160:147-151. 